Article

\title{
Anodic Activity of Hydrated and Anhydrous Iron (II) Oxalate in Li-Ion Batteries
}

\author{
Fatemeh Keshavarz $^{1, *}$, Marius Kadek ${ }^{2,3} \mathbb{D}$, Bernardo Barbiellini ${ }^{1,3} \mathbb{D}$ and Arun Bansil ${ }^{3}$ \\ 1 Department of Physics, School of Engineering Science, LUT University, FI-53851 Lappeenranta, Finland; \\ Bernardo.Barbiellini@lut.fi \\ 2 Hylleraas Centre for Quantum Molecular Sciences, UiT The Arctic University of Norway, \\ N-9037 Tromsø, Norway; Marius.Kadek@uit.no \\ 3 Department of Physics, Northeastern University, Boston, MA 02115, USA; Ar.Bansil@northeastern.edu \\ * Correspondence: Fatemeh.Keshavartz@lut.fi
}

check for updates

Citation: Keshavartz, F.; Kadek, M.; Barbiellini, B.; Bansil, A. Anodic Activity of Hydrated and Anhydrous Iron (II) Oxalate in Li-Ion Batteries. Condens. Matter 2022, 7, 8. https:// doi.org/10.3390/condmat7010008

Academic Editor: Víctor Manuel García Suárez

Received: 22 December 2021

Accepted: 10 January 2022

Published: 12 January 2022

Publisher's Note: MDPI stays neutral with regard to jurisdictional claims in published maps and institutional affiliations.

Copyright: (c) 2022 by the authors. Licensee MDPI, Basel, Switzerland. This article is an open access article distributed under the terms and conditions of the Creative Commons Attribution (CC BY) license (https:// creativecommons.org/licenses/by/ $4.0 /)$.

\begin{abstract}
We discuss the applicability of the naturally occurring compound Ferrous Oxalate Dihydrate (FOD) $\left(\mathrm{FeC}_{2} \mathrm{O}_{4} \cdot 2 \mathrm{H}_{2} \mathrm{O}\right)$ as an anode material in Li-ion batteries. Using first-principles modeling, we evaluate the electrochemical activity of FOD and demonstrate how its structural water content affects the intercalation reaction and contributes to its performance. We show that both $\mathrm{Li}^{0}$ and $\mathrm{Li}^{+}$ intercalation in FOD yields similar results. Our analysis indicates that fully dehydrated ferrous oxalate is a more promising anodic material with higher electrochemical stability: it carries $20 \%$ higher theoretical Li storage capacity and a lower voltage (0.68 V at the PBE/cc-pVDZ level), compared to its hydrated $(2.29 \mathrm{~V})$ or partially hydrated $(1.43 \mathrm{~V})$ counterparts
\end{abstract}

Keywords: Li-ion battery; metal-organic frameworks; iron oxalate; anode; electrochemical potential; water; first-principles calculations; density functional theory (DFT)

\section{Introduction}

Iron (II) oxalate dihydrate (Ferrous oxalate dihydrate; $\mathrm{FeC}_{2} \mathrm{O}_{4} \cdot 2 \mathrm{H}_{2} \mathrm{O}$; FOD) or humboldtine is a secondary mineral naturally found with lignite, pegmatite, and brown coal [1]. It can also be synthesized, for example, from hematite and oxalic acid [2]. FOD is known as one of the simplest coordination polymers (CPs) and a one-dimensional metal-organic framework (1D-MOF) [3,4]. Its structure is an extended chain made of $\mathrm{Fe}^{2+}$ metal nodes coordinated with two water molecules and oxalate organic linkers. When different FOD chains stack to form a crystal, they can create the $\alpha$-monoclinic or the $\beta$-orthorhombic allotropic form through extensive hydrogen bonding [5,6]. The $\alpha$ form can transform irreversibly into the $\beta$ form under suitable conditions [7].

Because of its high proton conductivity $\left(1.3 \mathrm{mS} \mathrm{cm}^{-1}\right)$ [8] and photocatalytic activity [3,9], FOD has been mainly used as an inexpensive material for photocatalytic applications [3]. Its extraordinary photocatalytic and Fenton activities have also motivated its application to wastewater treatment [4]. In addition, FOD has shown promising potential for the development of battery electrodes. Both anhydrous (AFO) and dihydrate (FOD) forms of ferrous oxalate have been recommended as promising Li-storage and anode materials for Li-ion batteries [10,11]. However, most studies have used FOD as a precursor for their electrode materials (for example [12-14]) because of its low thermal stability [15].

Although spectroscopic and structural properties of FOD are well-studied [1,3,4,15], studies of its electrochemical properties remain limited. In particular, the mechanism of $\mathrm{Li}$ intercalation into FOD and the corresponding electrochemical changes are unknown. To this end, we deploy first-principles calculations to model Li intercalation into FOD and its anhydrous form (AFO), as well as a partially hydrated ferrous oxalate structure (PHFO), and evaluate the associated electrochemical properties for use as an anode material. Notably, Fan et al. [3] has reported the electronic structure of a 1D FOD chain using density 
functional theory (DFT)-based calculations using the local density approximation (LDA) and a Hubbard U correction. Also, Zhang et al. [11] have proposed a mechanism for $\mathrm{Li}^{+}$ diffusion into periodic $\alpha$ and $\beta$-AFO crystals using the PBE-DFT method. However, no theoretical work has fully addressed the changes in the electrochemical properties of FOD upon dehydration and Li intercalation.

Our modeling shows that the structural water molecules of FOD hydrate the intercalating Li species, which enhances Li adsorption and increases the open-circuit voltage. In contrast, the fully dehydrated material yields a lower voltage that is favorable for anodic materials. Our analysis further indicates that $\mathrm{Li}^{0}$ or $\mathrm{Li}^{+}$intercalation in FOD yields similar results.

\section{Computational Method}

Note that FOD, AFO, and PHFO can be in the $\alpha$-monoclinic, $\beta$-orthorhombic, or mixed states $[6,11]$, and that their molecular and crystal structures are pressure dependent [15]. Bearing this in mind, we used a single chain of the three coordination polymers and ran the calculations on a subunit of their chain structure in order to focus on their intrinsic electrochemical properties rather than their structural features. The subunit contained three $\mathrm{Fe}^{2+}$ ions, two $\mathrm{C}_{2} \mathrm{O}_{4}{ }^{2-}$ bidentate anions bridging the metal ions and two or zero axial water molecules for each metal center. To balance the charge of the structures in accord with the coordination mode of the removed oxalate anions, each tail oxalate anion was represented by one $\mathrm{OH}^{-}$anion and one neutral $\mathrm{OH}$ group, which resulted in a neutral model. The orientation of the water molecules was adjusted along the lines of the study of Fan et al. [3]. The model structures are shown in Figure 1. The efficacy of the adopted models was further verified by comparison to experimental data and periodic calculations as discussed below and in the "Results and discussion" section. All model-based calculations were performed using the Gaussian 16 A.03 package [16].

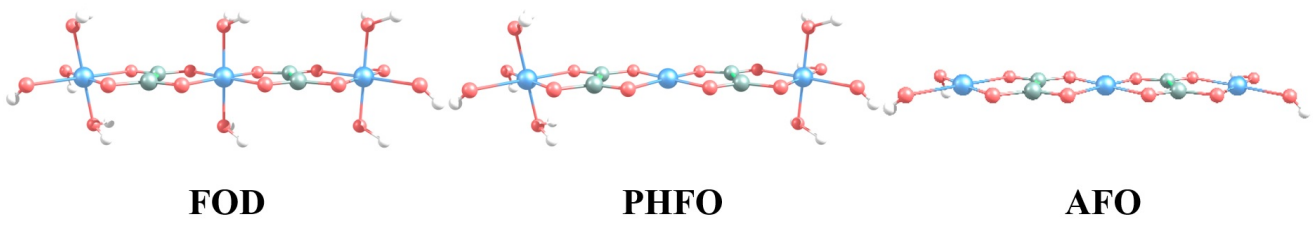

Figure 1. The starting models used to represent ferrous oxalate dihydrate (FOD), partially hydrated ferrous oxalate (PHFO), and anhydrous ferrous oxalate (AFO). Blue, red, grey, and white spheres represent the $\mathrm{Fe}$, oxygen, carbon, and hydrogen atoms, respectively.

As the properties of FOD are better understood compared with PHFO and AFO, we focused first on FOD. In order to determine the ground state of FOD, we carried out geometry and energy optimizations for various spin-dependent states using a variety of DFT-based approaches, including B3LYP [17], M06-L [18], PBE [19], PBE0 [19], and wB97XD [20], all using the cc-pVDZ basis set. The PBE method is of particular interest because it has been the function of choice in most existing studies on metal oxalates [11,21-24]. SVMN5 local-spin-density approximation [25] was also used in view of the success of such methods in investigating FOD [3]. Van der Waals interactions were included in all calculations using Grimme's D3 (GD3) semi-empirical dispersion correction [26] with the exception of computations based on the $\omega$ B97XD functional, which intrinsically supports long-range exchange-correlation corrections [20,27].

After identification of the ground state (Table S1 and Figure S1 of the Supporting Information), the computational level was calibrated by comparing the geometries and vibrational frequencies of the model FOD structure with the corresponding experimentally available results $[1,15]$. As seen from Table S2 and Section S1 (Supporting Information), PBE0/cc-pVDZ shows the smallest error in the modeled structures. Electrochemical potentials for FOD, AFO, and PHFO as anode materials were then calculated by inserting a $\mathrm{Li}^{+}$ion near the central $\mathrm{Fe}^{2+}$ atom (i.e., anodic reaction: $\mathrm{Fe}^{2+}+\mathrm{Li}^{+} \rightarrow \mathrm{Fe}^{3+}+\mathrm{Li}^{0}$ ) and 
optimizing the structures at the PBE0/cc-pVDZ level. Also, $\mathrm{Li}^{0}$ intercalation was modeled because the electron of $\mathrm{Li}^{0}$ is highly delocalized in solid systems, and modeling of $\mathrm{Li}$ intercalation into periodic crystals requires system neutrality. The anodic potential was also calculated at the PBE/cc-pVDZ, B3LYP/cc-pVDZ, and $\omega$ B97XD/cc-pVDZ levels for comparison purposes. Throughout the calculations, the stability of the structures was confirmed by the absence of any imaginary frequencies.

Our choice of the model was further cross-validated against 3D periodic boundary calculations on the unit cell of FOD [1]. For this purpose, we used the ReSpect (Relativistic Spectroscopy) package [28] developed for studies of molecular properties such as the nuclear magnetic and electron paramagnetic resonance parameters [29-31], as well as response to time-dependent electric fields [32-34]. This code recently enabled incorporating periodic boundary conditions together with Gaussian-type basis sets [35] that we used to calculate the electronic band structure and density of states of FOD (Figure 2) at the PBE/ucc-pVDZ level (" $u$ " indicates that the cc-pVDZ basis set was fully uncontracted). We employed a $\Gamma$-centered $5 \times 11 \times 7$ mesh of momentum-space points for the ground-state optimization procedure and a $15 \times 33 \times 21$ mesh for the calculation of the density of states.

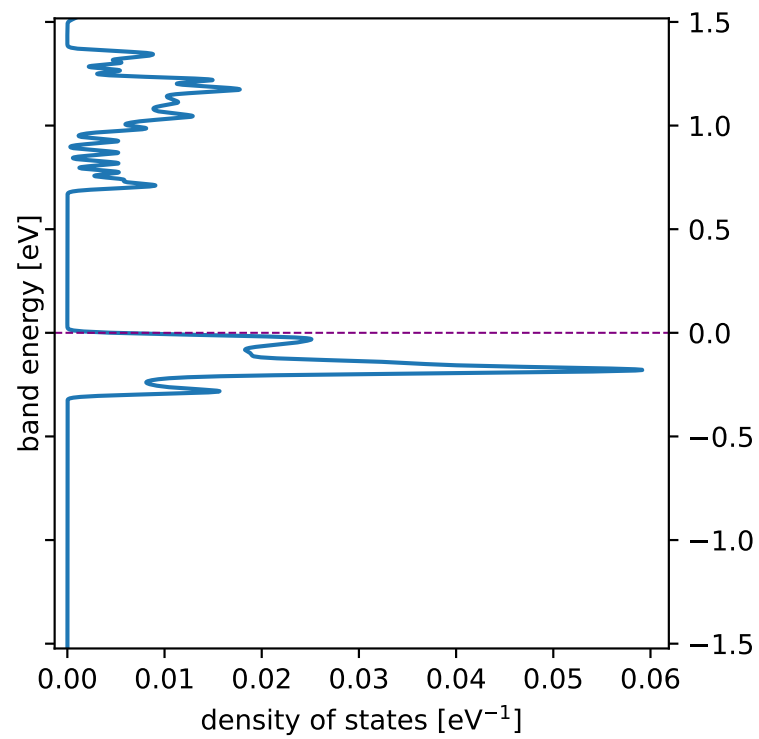

Figure 2. Electronic density of states (DOS) of ferrous oxalate dihydrate (FOD) based on using 3D periodic boundary conditions at the PBE/ucc-pVDZ level. The DOS was calculated using a dense mesh of $15 \times 33 \times 21$ momentum-space points and an artificial Gaussian broadening of $\sigma=0.01 \mathrm{eV}$. The dashed line indicates the Fermi level. The value of the bandgap calculated using the DOS is $0.7 \mathrm{eV}$.

\section{Results and Discussion}

\subsection{Spin State and Electronic Band Structure}

For the model-based calculations, the ground spin-state was unknown. Considering the presence of three $\mathrm{Fe}^{2+}$ ions in the models, various possibilities from singlet to 13tet were evaluated for the FOD model. The results of most computational levels in supplementary Figure S1 and Table S1 suggest that FOD is high spin (13tet) and its ground state has 1.0 to $24.4 \mathrm{~kJ} \mathrm{~mol}^{-1}(=0.01$ to $0.25 \mathrm{eV}$ ) energy difference with the first excited state (the 11tet state). At the PBE/cc-pVDZ and SVWN5/cc-pVDZ levels, the 11tet state is more stable than the 13tet state with $18.1 \mathrm{~kJ} \mathrm{~mol}^{-1}(=0.19 \mathrm{eV})$ and $43.6 \mathrm{~kJ} \mathrm{~mol}^{-1}(=0.45 \mathrm{eV})$ electronic energy difference, respectively. Regardless of the computational level, the 11tet state presents a curved structure, in contrast to the straight-chain nature of FOD in its crystals $[3,15]$. Furthermore, our results show a continuous decrease in FOD stability by shifting from the higher spin states to the lower ones. Particularly, the singlet state calculations at most computational levels failed to converge, indicating that the singlet state should be an 
excited state as the convergence of highly excited states is challenging when DFT methods are applied. The 13tet and singlet states differ by over $5 \mathrm{eV}$ in energy.

Beyond the model-based calculations, the electronic structure of FOD was obtained by running full periodic calculations on its unit cell at the PBE/ucc-pVDZ level, which yielded total energy of $-195,183.57 \mathrm{eV}$ per unit cell. The model-based calculations at the $\mathrm{PBE} / \mathrm{cc}-\mathrm{pVDZ}$ and PBE/ucc-pVDZ levels, respectively, gave the total electronic energies of $-144,367.79 \mathrm{eV}$ and $-144,372.09 \mathrm{eV}$, including the zero-point energy (ZPE) correction. Figure 2 shows the density of states obtained from the periodic calculation with a bandgap of $0.7 \mathrm{eV}$. The vertical gap at the $\Gamma$ point is $0.94 \mathrm{eV}$ indicating that FOD is an indirect semiconductor. Notably, the periodic calculations of Fan et al. using Ceperley-Alder localdensity approximation with plane-wave basis set and a Hubbard-U correction [3] found a direct bandgap of $1.13 \mathrm{eV}$ in FOD, while our model-based PBE/cc-pVDZ (PBE/ucc-pVDZ) calculations estimate the bandgap to be $1.36(1.42) \mathrm{eV}$ and the experimentally determined band gaps are $2.10 \mathrm{eV}$ [4] and $2.17 \mathrm{eV}$ [36]. Although DFT-based methods are well known to underestimate the bandgap in semiconductors [37], our model-based PBE/cc-pVDZ and PBE/ucc-pVDZ calculations reproduce the electronic properties of FOD reasonably. Since our model-based PBE/ucc-pVDZ calculations were affected by the poor quadrature accuracy, we expect the PBE/cc-pVDZ results to be more reliable.

\subsection{Intercalation Mechanism}

The anodic response of FOD, PHFO, and AFO depends on the mechanism of Li diffusion and intercalation. The mechanism of $\mathrm{Li}^{+}$diffusion into the channels of AFO is explained by Zhang et al. [11], who reported a value of $3.11 \times 10^{-10} \mathrm{~cm}^{2} \mathrm{~s}^{-1}$ for anodic diffusion of $\mathrm{Li}^{+}$into $\alpha @ \beta-\mathrm{FeC}_{2} \mathrm{O}_{4}$. They argue that $\mathrm{Li}^{+}$can diffuse through the short diffusion channels created between the AFO chains [11], which show low resistance against $\mathrm{Li}^{+}$ diffusion. However, fast transmission of $\mathrm{Li}^{+}$at higher cycling rates can lead to irreversible structural defects [11]. Moreover, they deduced from their modeling and experimental results that $\mathrm{Li}^{+}$ions occupy the primary sites of water molecules or they just diffuse bilaterally along the chains (the hydrogen bonding network between the two chains) and hop between the layers [11]. Such a diffusion mechanism is at play in the $\mathrm{Li}^{0}$ and $\mathrm{Li}^{+}$ intercalated structures shown in Figures 3, S2, and S3. Based on the obtained structures, both $\mathrm{Li}^{0}$ and $\mathrm{Li}^{+}$prefer binding to the oxalate oxygens of PHFO and $\mathrm{AFO}$, consistent with the bilateral diffusion mode. In the case of FOD, the $\mathrm{Li}^{0}$ and $\mathrm{Li}^{+}$intercalation mechanisms involve the replacement of an axial water molecule, subsequent modification of the hydrogen bonding network, and induction of curvature in the straight-chain structure. This intercalation model suggests hydration of Li by the structural FOD water molecules. Reversible and irreversible structural changes induced by Li intercalation have also been reported for other Fe-containing electrodes [38,39].

To ascertain that the changes in the hydrogen bonding and chain curvature of FOD are not an artifact of the chosen modeling parameters, the starting FOD structure (Figure 1) was optimized in the presence of $\mathrm{Li}^{+}$at both PBE/cc-pVDZ and PBE0/cc-pVDZ levels by considering two possibilities: (a) FOD structure being fully flexible, and (b) the edge $\mathrm{Fe}^{2+}$ ions and their attached water and hydroxyl groups being fixed in their positions. The results (Figure S4) confirm that water displacement/ $\mathrm{Li}^{+}$hydrolysis is the key to intercalation of $\mathrm{Li}^{+}$into $\mathrm{FOD}$, and hydrogen-bonding-network modification and curvature induction are inevitable. Furthermore, the results demonstrate that the conservation of the crystal structure of FOD, PHFO, and AFO (straight ferrous oxalate chains) depends on the presence of axial water molecules or intercalated Li. 

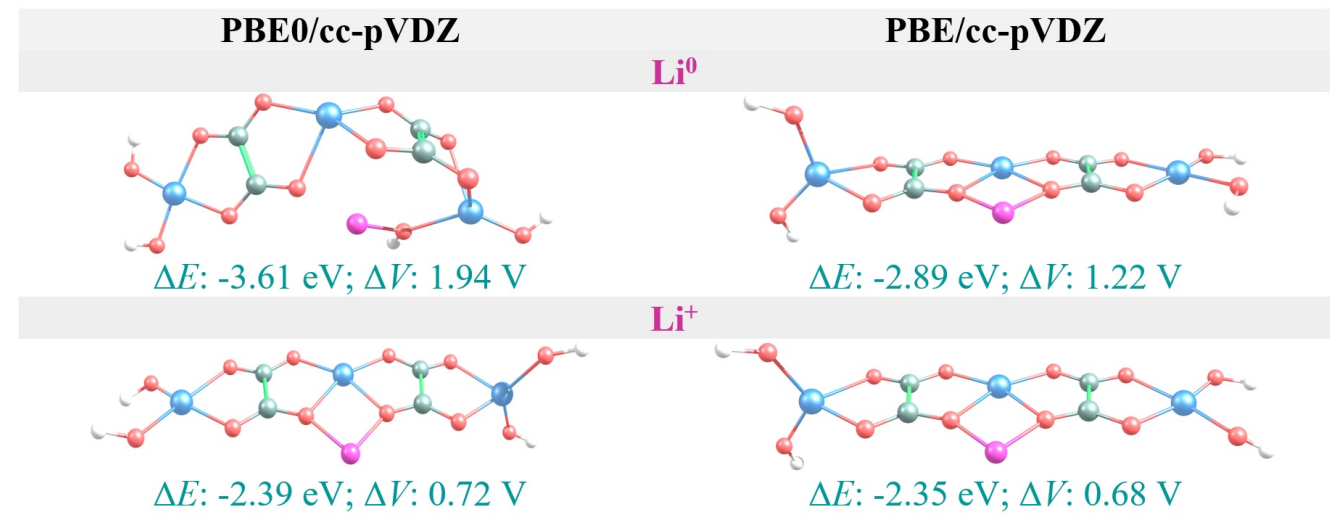

Figure 3. AFO structure after Li intercalation and the corresponding adsorption energy (zero-point energy corrected; $\Delta E$ ) and open-circuit voltage with respect to Li cathode $(\Delta V)$. The pink, blue, red, grey, and white spheres represent the $\mathrm{Li}, \mathrm{Fe}$, oxygen, carbon, and hydrogen atoms, respectively.

Natural bond-orbital (NBO) analysis was performed at the PBE0/cc-pVDZ level to identify the most important interactions involved in Li intercalation. Accordingly, charge transfer from the lone-pair (LP) orbitals of oxalate oxygen atoms to the anti-LP (LP*) orbital of $\mathrm{Li}$ is the only significant driver of $\mathrm{Li}$ intercalation into PHFO and AFO with stabilization energy of over $0.10 \mathrm{eV}$. In the case of FOD, charge transfer from the LP orbital of a water molecule's oxygen atom to the $\mathrm{LP}^{*}$ orbital of $\mathrm{Li}$ is the most important contributor to the intercalation process. This highlights the role of Li hydration in the electrochemical reaction.

\subsection{Electrochemical Potential}

To evaluate the anodic potential, both the Li adsorption energy $(\Delta E)$ and the opencircuit voltage $(\mathrm{OCV} ; \Delta V)$ were calculated using [40,41]:

$$
\begin{gathered}
\Delta E=E_{F O / x L i}-E_{F O}-x E_{L i} \\
\Delta V=-\left(E_{F O / x L i}-E_{F O}-x E_{L i-b u l k}\right) / x n e
\end{gathered}
$$

where FO represents FOD, PHFO or AFO, and $E_{F O}$ is the energy of Li-free FO. $E_{F O / x L i}, E_{L i}$ and $E_{L i-b u l k}$ are the total energies of the $\mathrm{Li}^{0}$ or $\mathrm{Li}^{+}$intercalated $\mathrm{FO}, \mathrm{Li}^{0}$ atom or $\mathrm{Li}^{+}$ion and bulk Li metal, respectively. $x$ is the number of intercalated $\mathrm{Li}^{0}$ atoms or $\mathrm{Li}^{+}$ions $(=1), n$ is the highest oxidation state of the intercalated $\mathrm{Li}(=1)$ and $e$ is electron charge. To obtain $E_{L i-b u l k}$, the cohesion energy of Li metal $\left(E_{c o h}\right)$ and the energy of Li atom $\left(E_{L i}\right)$ were used as follows [42,43].

$$
\Delta E_{c o h}=E_{L i}-E_{L i-b u l k}=1.65 \mathrm{eV}
$$

Based on the values reported in Figures 1 and S2-S4, the intercalation of Li into the three ferrous oxalate models is favorable, but the adsorption potency decreases with the increase of water content of ferrous oxalate. OCV increases with the increase of the hydration level. For anodes, lower the OCV, better the battery performance [44]. Therefore, AFO would be a better anode material compared to FOD, and PHFO would display an intermediate efficiency. This finding is in line with a recent study of the effect of crystal water on the anodic efficiency of FOD [45], which finds that water molecules inhibit the electrochemical activity of ferrous oxalate by creating structural deficiencies. Our NBO results and the FOD structural transformations shown in Figure S4, also show that the structural water molecules hydrate $\mathrm{Li}$, cause structural deficiencies, and inhibit the anodic potential of ferrous oxalate. Moreover, our results of Table 1 and Figures S5 and S6, indicate that the presence of water has two roles: (1) it affects the charge distribution of ferrous oxalate-higher the water content, lower the positive charge localized on the Fe ions, and (2) it lowers the energy levels of the ferrous-oxalate molecular orbitals and decreases the 
bandgap of AFO. The DOS graphs also indicate noticeable changes in the density of states of the ferrous oxalate models upon Li intercalation.

Table 1. Electronic properties and atomic charges of FOD, PHFO and AFO before and after Li intercalation, at the PBE0/cc-pVDZ level ${ }^{a}$.

\begin{tabular}{cccccccc}
\hline Structure & $E_{\text {НОМо }}$ & $E_{\text {LUМO }}$ & $E_{g}$ & $E_{F}$ & $C_{F e}$ & $C_{F e, \text { total }}$ & $C_{L i}$ \\
\hline FOD & -6.86 & -1.96 & 4.90 & -4.41 & 1.03784 & 3.23523 & - \\
PHFO & -7.18 & -2.44 & 4.74 & -4.81 & 1.25910 & 3.45593 & - \\
AFO & -8.37 & -3.29 & 5.08 & -5.83 & 1.29025 & 3.86257 & - \\
$\mathrm{Li}^{+} /$FOD & -9.66 & -4.50 & 5.17 & -7.08 & 1.16869 & 3.50852 & 0.90070 \\
$\mathrm{Li}^{+} /$FOD & -9.68 & -5.03 & 4.65 & -7.35 & 1.06932 & 3.30792 & 0.92476 \\
$\mathrm{Li}^{+} /$PHFO & -9.92 & -5.21 & 4.71 & -7.57 & 1.24936 & 3.44727 & 0.94548 \\
$\mathrm{Li}^{+} /$AFO & -11.07 & -6.29 & 4.77 & -8.68 & 1.28599 & 3.89290 & 0.95229 \\
$\mathrm{Li}^{0} /$ FOD & -7.15 & -1.75 & 5.40 & -4.45 & 1.16988 & 3.48206 & 0.89520 \\
$\mathrm{Li}^{0} /$ PHFO & -6.43 & -1.90 & 4.53 & -4.17 & 1.19598 & 3.46516 & 0.89973 \\
$\mathrm{Li}^{0} /$ AFO & -6.77 & -3.73 & 3.04 & -5.25 & 1.24761 & 3.53108 & 0.90271
\end{tabular}

${ }^{a} E_{\text {HOMO }}(\mathrm{eV})$ : Energy of the highest occupied molecular orbital; $E_{L U M O}(\mathrm{eV})$ : energy of the lowest unoccupied molecular orbital; $E_{g}(\mathrm{eV})$ : band gap energy $\left(=E_{L U M O}-E_{\text {HOMO }}\right) ; E_{F}(\mathrm{eV})$ : Fermi level energy $\left(=\left(E_{\text {LUMO }}+\right.\right.$ $\left.\left.E_{\mathrm{HOMO}}\right) / 2\right) ; C_{\mathrm{Fe}}(\mathrm{C})$ : atomic charge of the central Fe ion based on a according to natural bond-orbital analysis; $C_{F e, \text { total }}(\mathrm{C})$ : total atomic charges of the three Fe ions according to natural bond-orbital analysis; and $C_{L i}(\mathrm{C})$ : atomic charge of $\mathrm{Li}$ according to natural bond-orbital analysis. ${ }^{b}$ FOD* refers to the properties obtained by considering the geometry of the edge $\mathrm{Fe}^{2+}$ ions and their hydroxyl groups and water molecules fixed (rigid).

Considering $\mathrm{Li}^{0}$ intercalation, the OCVs of $\mathrm{AFO}, \mathrm{PHFO}$, and FOD at the PBE/cc-pVDZ (and PBE0/cc-pVDZ) level/s were calculated to be $1.22 \mathrm{~V}(1.94 \mathrm{~V}), 1.34 \mathrm{~V}(3.13 \mathrm{~V})$, and $2.85 \mathrm{~V}(3.58 \mathrm{~V})$, respectively, see Table 2 and Figure S3. Zhang et al. reported the reducing and oxidating peaks of $0.75 \mathrm{~V}$ and $1.5 \mathrm{~V}$ for their $\alpha @ \beta-\mathrm{FeC}_{2} \mathrm{O}_{4}$ electrode, respectively [11]. Their anodic voltage $(1.5 \mathrm{~V})$ agrees with that of PHFO, which lacks the axial water molecules of its central $\mathrm{Fe}^{2+}$ ion, but it is higher than our predicted AFO voltage. This clarifies the impact of hydration level and crystalline structure on the electrochemical activity of the ferrous oxalate family and recommends the application of fully dehydrated ferrous oxalate as an anode material.

Table 2. Computed adsorption energies $(\Delta E ; \mathrm{eV})$ and open-circuit voltages $(\mathrm{OCV} ; \Delta V ; \mathrm{V})$ for $\mathrm{Li}^{+}$and $\mathrm{Li}^{0}$ intercalation.

\begin{tabular}{ccccc}
\hline & \multicolumn{2}{c}{ PBE0/cc-pVDZ } & \multicolumn{2}{c}{ PBE/cc-pVDZ } \\
\hline & \multicolumn{3}{c}{$\mathrm{Li}^{+}$intercalation } & $\Delta E$ \\
\hline FDO & $\Delta E$ & $\Delta V$ & -3.96 & 2.29 \\
PHFO & -4.40 & 2.73 & -3.10 & 1.43 \\
AFO & -3.14 & 1.47 & -2.35 & 0.68 \\
\hline & -2.39 & 0.72 & & \\
\hline & \multicolumn{5}{c}{$\mathrm{Li}^{0}$ intercalation } & $\Delta E$ \\
FDO & $\Delta E$ & $\Delta V$ & -4.52 & 2.85 \\
AFO & -5.25 & 3.58 & -3.01 & 1.34 \\
& -4.80 & 3.13 & -2.89 & 1.22 \\
\hline
\end{tabular}

Theoretical adsorption capacities $(A C)$ of the ferrous oxalate models $(\mathrm{FO})$ were calculated by using [41]

$$
A C=x n F /\left(M W_{F O / x L i}\right)
$$


Here, $F\left(=26,801 \mathrm{mAh} \mathrm{mol}^{-1}\right)$ is the Faraday constant, and $M W_{F O / x L i}$ is the molecular weight of the Li containing ferrous oxalate models. Since we have not modeled intercalation of multiple Li species to obtain the maximum number of Li species that can be intercalated $(x)$, we have considered the case where each model structure only accommodates one $\mathrm{Li}$ atom/ion. This gave the $A C$ values of $64.00,54.60$ and $50.87 \mathrm{mAh} \mathrm{g}^{-1}$ for AFO, PHFO, and FOD, respectively. These $A C$ values indicate that AFO has the highest capacity for application as an anode in Li-ion batteries. AFO is also associated with higher longterm stability [45]. Our $A C$ trend showing fully dehydrated ferrous oxalate with about $20 \%$ higher adsorption capacity agrees with experimental measurements $[10,45]$. Clearly, the actual value of the capacity would be higher if more than one $\mathrm{Li}$ atom/ion can be accommodated in the model structure.

\section{4. $\mathrm{Li}^{+}$vs. $\mathrm{Li}^{0}$ Intercalation}

Electrochemical performance of AFO, PHFO, and FOD was assessed by modeling the intercalation of $\mathrm{Li}^{0}$ as well as $\mathrm{Li}^{+}$. The choice of $\mathrm{Li}^{0}$ is in accord with the general practice of system neutrality and delocalization of Li charge in periodic structures. The choice of $\mathrm{Li}^{+}$was motivated by the expected electron transfer reaction $\left(\mathrm{Fe}^{2+}+\mathrm{Li}^{+} \rightarrow \mathrm{Fe}^{3+}+\mathrm{Li}^{0}\right)$. The results summarized in Table 2 indicate that both choices yield similar performance and demonstrate the inhibitory effect of structural water and the higher anodic efficiency of AFO. Figures 1 and S2-S6, and Table 1 further show the insensitivity of our qualitative results to the computational level and Li charge state used in the computations.

\section{Conclusions}

Using DFT-based simulations, we demonstrate the mechanism of Li intercalation in ferrous oxalate materials with different structural water contents. The electrochemical activity of the materials for application to Li-ion battery anodes is evaluated. Our analysis indicates that the presence of water decreases the capacity of ferrous oxalate for Li intercalation and increases the resulting voltage, and degrades its performance as an anode by inhibiting its electrochemical activity.

Supplementary Materials: The following supporting information can be downloaded at: https: / / www.mdpi.com/article/10.3390/condmat7010008/s1, Section S1: Computational level validation; Figure S1: Geometry of FOD optimized for different spin states at various computational levels; Figures S2 and S3: Changes in the structure of FOD, PHFO and AFO upon $\mathrm{Li}^{+} / \mathrm{Li}^{0}$ intercalation and the corresponding adsorption energies and open circuit voltages; Figure S4: The structure of FOD after $\mathrm{Li}^{+}$intercalation and the corresponding adsorption energy and the open-circuit voltage; Figures S5 and S6: Changes in the density of states (DOS) of FOD, PHFO and AFO upon Li $/ \mathrm{Li}^{0}$ intercalation; Table S1: Zero-point energy corrected electronic energy, Gibbs free energy and the total spin of FOD before and after annihilation in different spin states for various computational levels; Table S2: Comparison of the geometry of the FOD model (13tet) optimized at different levels of theory with the experimental structure.

Author Contributions: Conceptualization, B.B.; Formal analysis, F.K. and M.K.; Funding acquisition, A.B.; Investigation, F.K. and M.K.; Project administration, B.B.; Resources, B.B. and A.B.; Supervision, B.B.; Writing—original draft, F.K. and M.K.; Writing—review \& editing, B.B. and A.B. All authors have read and agreed to the published version of the manuscript.

Funding: The work at LUT university was supported by the project Puhdas Ilma from the PROFI 5 support of the Academy of Finland (Grant no. 326325). The work at Northeastern University was supported by the U.S. Department of Energy (DOE), Office of Science, Basic Energy Sciences Grant No. DE- FG02-07ER46352, and the NERSC supercomputing center through DOE Grant No. DE-AC02-05CH11231. M.K. acknowledges the support of the Research Council of Norway through its Centres of Excellence scheme (Grant No. 262695) and its Mobility Grant scheme (Grant No. 301864), as well as the Norwegian Supercomputer Program NOTUR through a grant for computer time (Grant No. NN4654K). 
Data Availability Statement: The data supporting the findings of this study are freely available upon request from the corresponding author.

Acknowledgments: The authors acknowledge CSC-IT Center for Science (Finland) and Northeastern University's Advanced Scientific Computation Center(ASCC) for computational resources.

Conflicts of Interest: The authors declare no conflict of interest.

\section{References}

1. Echigo, T.; Kimata, M. Single-crystal X-ray diffraction and spectroscopic studies on humboldtine and lindbergite: Weak Jahn-Teller effect of $\mathrm{Fe}^{2+}$ ion. Phys. Chem. Miner. 2008, 35, 467-475. [CrossRef]

2. Vehmaanperä, P.; Gong, B.; Sit, P.H.L.; Salmimies, R.; Barbiellini, B.; Häkkinen, A. Formation of humboldtine during the dissolution of hematite in oxalic acid-density functional theory (DFT) calculations and experimental verification. Clays Clay Miner. 2021. [CrossRef]

3. Fan, X.; Zhang, L.; Li, M.; Wang, M.; Zhou, X.; Cheng, R.; Zhou, Y.; Shi, J. $\alpha$-Ferrous oxalate dihydrate: A simple coordination polymer featuring photocatalytic and photo-initiated Fenton oxidations. Sci. China Mater. 2016, 59, 574-580. [CrossRef]

4. Li, K.; Liang, Y.; Yang, J.; Yang, G.; Xu, R.; Xie, X. $\alpha$-Ferrous oxalate dihydrate: An Fe-based one-dimensional metal organic framework with extraordinary photocatalytic and Fenton activities. Catal. Sci. Technol. 2018, 8, 6057-6061. [CrossRef]

5. Deyrieux, R.; Peneloux, A. Studies on some divalent metal oxalates. I. Crystal structure of 2 allotropic forms of dihydrated ferrous oxalate. Bull. Soc. Chim. Fr. 1969, 8, 2675.

6. Śledzińska, I.; Murasik, A.; Piotrowski, M. Neutron diffraction study of crystal and magnetic structures of $\alpha-\mathrm{FeC}_{2} \mathrm{O}_{4} \cdot 2 \mathrm{D}_{2} \mathrm{O}$. Phys. $B+C$ 1986, 138, 315-322. [CrossRef]

7. Lagier, J.; Pezerat, H. Etude des phases obtenues lors de la preparation doxalates de metaux de transition. Comptes Rendus Hebd. Des Seances Acad. Des Sci. Ser. 1967, 264, 496.

8. Yamada, T.; Sadakiyo, M.; Kitagawa, H. High proton conductivity of one-dimensional ferrous oxalate dihydrate. J. Am. Chem. Soc. 2009, 131, 3144-3145. [CrossRef]

9. Liu, Z.J.; Liu, W.; Wang, Y.; Guo, M.L. Preparation of $\beta$-ferrous oxalate dihydrate layered nanosheets by mechanochemical method and its visible-light-driven photocatalytic performance. Mater. Lett. 2016, 178, 83-86. [CrossRef]

10. Aragón, M.J.; León, B.; Perez Vicente, C.; Tirado, J.L. Synthesis and electrochemical reaction with lithium of mesoporous iron oxalate nanoribbons. Inorg. Chem. 2008, 47, 10366-10371. [CrossRef]

11. Zhang, K.; Li, Y.; Wang, Y.; Zhao, J.; Chen, X.; Dai, Y.; Yao, Y. Enhanced electrochemical properties of iron oxalate with more stable $\mathrm{Li}^{+}$ions diffusion channels by controlling polymorphic structure. Chem. Eng. J. 2020, 384, 123281. [CrossRef]

12. Ojczyk, W.; Marzec, J.; Świerczek, K.; Zajac, W.; Molenda, M.; Dziembaj, R.; Molenda, J. Studies of selected synthesis procedures of the conducting LiFePO4-based composite cathode materials for Li-ion batteries. J. Power Sources 2007, 173, 700-706. [CrossRef]

13. Li, M.; Wang, W.; Yang, M.; Lv, F.; Cao, L.; Tang, Y.; Sun, R.; Lu, Z. Large-scale fabrication of porous carbon-decorated iron oxide microcuboids from Fe-MOF as high-performance anode materials for lithium-ion batteries. RSC Adv. 2015, 5, 7356-7362. [CrossRef]

14. Wang, C.; Wang, R.; Peng, Y.; Chen, J.; Chen, Z.; Yin, H.; Li, J. Nb-incorporated Fe (oxy) hydroxide derived from structural transformation for efficient oxygen evolution electrocatalysis. J. Mater. Chem. A 2020, 8, 24598-24607. [CrossRef]

15. Müller, H.; Bourcet, L.; Hanfland, M. Iron (II) oxalate dihydrate-Humboldtine: Synthesis, spectroscopic and structural properties of a versatile precursor for high pressure research. Minerals 2021, 11, 113. [CrossRef]

16. Frisch, M.; Trucks, G.; Schlegel, H.; Scuseria, G.; Robb, M.; Cheeseman, J.; Scalmani, G.; Barone, V.; Petersson, G.; Nakatsuji, H.; et al. Gaussian 16 Revision a. 03. 2016; Gaussian Inc.: Wallingford, CT, USA, 2016; Volume 2.

17. Beck, A.D. Density-functional thermochemistry. III. The role of exact exchange. J. Chem. Phys. 1993, 98, 5648. [CrossRef]

18. Zhao, Y.; Truhlar, D.G. The M06 suite of density functionals for main group thermochemistry, thermochemical kinetics, noncovalent interactions, excited states, and transition elements: Two new functionals and systematic testing of four M06-class functionals and 12 other functionals. Theor. Chem. Acc. 2008, 120, 215-241.

19. Perdew, J.P.; Burke, K.; Ernzerhof, M. Generalized gradient approximation made simple. Phys. Rev. Lett. 1996, 77, 3865. [CrossRef]

20. Chai, J.D.; Head-Gordon, M. Long-range corrected hybrid density functionals with damped atom-atom dispersion corrections. Phys. Chem. Chem. Phys. 2008, 10, 6615-6620. [CrossRef]

21. Koleżyński, A.; Handke, B.; Drożdż-Cieśla, E. Crystal structure, electronic structure, and bonding properties of anhydrous nickel oxalate. J. Therm. Anal. Calorim. 2013, 113, 319-328. [CrossRef]

22. Colmenero, F.; Timón, V. Extreme negative mechanical phenomena in the zinc and cadmium anhydrous metal oxalates and lead oxalate dihydrate. J. Mater. Sci. 2020, 55, 218-236. [CrossRef]

23. Colmenero, F. Silver oxalate: Mechanical properties and extreme negative mechanical phenomena. Adv. Theory Simul. 2019, 2, 1900040. [CrossRef]

24. South, C.J.; Roy, L.E. Insights into the thermal decomposition of plutonium (IV) oxalate-a DFT study of the intermediate structures. J. Nucl. Mater 2021, 549, 152864. [CrossRef]

25. Vosko, S.H.; Wilk, L.; Nusair, M. Accurate spin-dependent electron liquid correlation energies for local spin density calculations: a critical analysis. Can. J. Phys. 1980, 58, 1200-1211. [CrossRef] 
26. Grimme, S.; Antony, J.; Ehrlich, S.; Krieg, H. A consistent and accurate ab initio parametrization of density functional dispersion correction (DFT-D) for the 94 elements H-Pu. J. Chem. Phys. 2010, 132, 154104. [CrossRef] [PubMed]

27. Singh, D.K.; Rathke, B.; Kiefer, J.; Materny, A. Molecular structure and interactions in the ionic liquid 1-ethyl-3-methylimidazolium trifluoromethanesulfonate. J. Phys. Chem. A 2016, 120, 6274-6286. [CrossRef]

28. Repisky, M.; Komorovsky, S.; Kadek, M.; Konecny, L.; Ekström, U.; Malkin, E.; Kaupp, M.; Ruud, K.; Malkina, O.L.; Malkin, V.G. ReSpect: Relativistic spectroscopy DFT program package. J. Chem. Phys. 2020, 152, 184101. [CrossRef] [PubMed]

29. Komorovskỳ, S.; Repiskỳ, M.; Malkina, O.L.; Malkin, V.G.; Malkin Ondík, I.; Kaupp, M. A fully relativistic method for calculation of nuclear magnetic shielding tensors with a restricted magnetically balanced basis in the framework of the matrix Dirac-Kohn-Sham equation. J. Chem. Phys. 2008, 128, 104101. [CrossRef]

30. Repiskỳ, M.; Komorovskỳ, S.; Malkina, O.L.; Malkin, V.G. Restricted magnetically balanced basis applied for relativistic calculations of indirect nuclear spin-spin coupling tensors in the matrix Dirac-Kohn-Sham framework. Chem. Phys. 2009, 356, 236-242. [CrossRef]

31. Repiskỳ, M.; Komorovskỳ, S.; Malkin, E.; Malkina, O.L.; Malkin, V.G. Relativistic four-component calculations of electronic g-tensors in the matrix Dirac-Kohn-Sham framework. Chem. Phys. Lett. 2010, 488, 94-97. [CrossRef]

32. Kadek, M.; Konecny, L.; Gao, B.; Repisky, M.; Ruud, K. X-ray absorption resonances near L 2, 3-edges from real-time propagation of the Dirac-Kohn-Sham density matrix. Phys. Chem. Chem. Phys. 2015, 17, 22566-22570. [CrossRef] [PubMed]

33. Konecny, L.; Kadek, M.; Komorovsky, S.; Ruud, K.; Repisky, M. Resolution-of-identity accelerated relativistic two-and fourcomponent electron dynamics approach to chiroptical spectroscopies. J. Chem. Phys. 2018, 149, 204104. [CrossRef] [PubMed]

34. Konecny, L.; Repisky, M.; Ruud, K.; Komorovsky, S. Relativistic four-component linear damped response TDDFT for electronic absorption and circular dichroism calculations. J. Chem. Phys. 2019, 151, 194112. [CrossRef]

35. Kadek, M.; Repisky, M.; Ruud, K. All-electron fully relativistic Kohn-Sham theory for solids based on the Dirac-Coulomb Hamiltonian and Gaussian-type functions. Phys. Rev. B 2019, 99, 205103. [CrossRef]

36. Fan, X.; Zhang, L.; Cheng, R.; Wang, M.; Li, M.; Zhou, Y.; Shi, J. Construction of graphitic $\mathrm{C}_{3} \mathrm{~N}_{4}$-based intramolecular donoracceptor conjugated copolymers for photocatalytic hydrogen evolution. ACS Catal. 2015, 5, 5008-5015. [CrossRef]

37. Hafner, J. Ab-initio simulations of materials using VASP: Density-functional theory and beyond. J. Comput. Chem. 2008, 29, 2044-2078. [CrossRef]

38. Combelles, C.; Yahia, M.B.; Pedesseau, L.; Doublet, M.L. Design of electrode materials for lithium-ion batteries: the example of metal- organic frameworks. J. Phys. Chem. C 2010, 114, 9518-9527. [CrossRef]

39. Keshavarz, F.; Kadek, M.; Barbiellini, B.; Bansil, A. Electrochemical Potential of the Metal Organic Framework MIL-101 (Fe) as Cathode Material in Li-Ion Batteries. Condens. Matter 2021, 6, 22. [CrossRef]

40. Tang, Q.; Zhou, Z.; Shen, P. Are MXenes promising anode materials for Li ion batteries? Computational studies on electronic properties and Li storage capability of $\mathrm{Ti}_{3} \mathrm{C}_{2}$ and $\mathrm{Ti}_{3} \mathrm{C}_{2} \mathrm{X}_{2}(\mathrm{X}=\mathrm{F}, \mathrm{OH})$ monolayer. J. Am. Chem. Soc. 2012, 134, 16909-16916. [CrossRef]

41. Lv, X.; Li, F.; Gong, J.; Gu, J.; Lin, S.; Chen, Z. Metallic FeSe monolayer as an anode material for Li and non-Li ion batteries: A DFT study. Phys. Chem. Chem. Phys. 2020, 22, 8902-8912. [CrossRef]

42. Zhou, F.; Cococcioni, M.; Marianetti, C.A.; Morgan, D.; Ceder, G. First-principles prediction of redox potentials in transition-metal compounds with LDA+ U. Phys. Rev. B 2004, 70, 235121. [CrossRef]

43. Callaway, J.; Zou, X.; Bagayoko, D. Total energy of metallic lithium. Phys. Rev. B 1983, 27, 631. [CrossRef]

44. Qian, L.; Yu, T.; Wei, Z.; Chang, B.; Huang, G.; Wang, Z.; Liu, Y.; Sun, H.; Bai, L.; Huang, W. Lower-voltage plateau Zn-substituted $\mathrm{Co}_{3} \mathrm{O}_{4}$ submicron spheres anode for Li-ion half and full batteries. J. Alloys Compd. 2022, 890, 161888. [CrossRef]

45. Zhang, K.; Li, Y.; Hu, X.; Liang, F.; Wang, L.; Xu, R.; Dai, Y.; Yao, Y. Inhibitive role of crystal water on lithium storage for multilayer $\mathrm{FeC}_{2} \mathrm{O}_{4} \cdot \mathrm{xH}_{2} \mathrm{O}$ anode materials. Chem. Eng. J. 2021, 404, 126464. [CrossRef] 